\title{
Clinical Effect of Naomaitong in Treating Cerebrovascular Diseases
}

\author{
Wentao Zhang ${ }^{1, a}$, Ping Zuo ${ }^{1}$, Feng Wang ${ }^{1}$, Yinglin Cui ${ }^{1}$ \\ ${ }^{1}$ Henan Provincial Hospital, Zhengzhou, Henan, 450002
}

\begin{abstract}
Objective: To explore the clinical effect of applying Naomaitong in the treatment of cerebrovascular diseases.

Method: The research work was carried out in our hospital from November 2018 to November 2019. A total of 100 patients were selected in this study, all of whom were cerebrovascular disease patients, and they were divided into two groups on average. Naofukang injection was named as the control group, and the other group was given Naofukang injection combined with Naomaitong oral liquid, and the therapeutic effects of the two groups were compared and analyzed.

Results: The treatment efficiency of the patients in the test group and the control group were $94.00 \%$ and $82.00 \%$, respectively. The effectiveness in the test group was higher. The difference in FIB and PagT levels between the two groups before treatment was small, $\mathrm{P}>0.05$, which was non-significant. After the intervention, the FIB and PagT levels in the test group were lower, and the improvement effect was more significant. The difference between the two groups was $\mathrm{P}<0.05$, which was significant.Conclusion: The application of Naomaitong therapy in the treatment of cerebrovascular diseases has a significant effect. It can reduce the incidence of adverse reactions in patients and improve the clinical symptoms of patients, which has a positive significance for clinical development.
\end{abstract}

\section{Introduction}

As far as cerebrovascular disease is concerned, it mainly includes cerebral infarction, cerebral hemorrhage and cerebral thrombosis, etc., and the clinical incidence is relatively high [1]. Cerebrovascular diseases have a higher mortality rate, a higher morbidity rate, and a higher disability rate, which has a serious impact on the quality of life and health of patients [2]. Therefore, it is necessary to carry out clinical and active treatment work. In this study, Naomaitong was applied to explore its clinical effect.

\section{Research objective}

By grouping, one group of patients were given Naofukang injection, and the group were added Naomaitong oral liquid on this basis to analyze the treatment effect of patients after intervention to clarify the intervention effect of Naomaitong in the treatment of cerebrovascular diseases.

\section{Patients data and research methods}

\subsection{Research materials}

In order to explore the application of Naomaitong in cerebrovascular diseases, we selected patients with cerebrovascular diseases who were treated in our hospital from November 2018 to November 2019. The number of patients was 100 . They were divided into two groups: one group was given Naofukang injection as control group, which had 50 people including 24 male and 26 female. The patient's age range was 54-78 years old and the patient's median age was $(61.05 \pm 5.45)$ years old. The other group was given Naofukang injection combined with Naomaitong oral liquid as test group, which also had 50 patients, including 25 males and 25 females. The patients' age range was from 55 to 77 years, with an average age of $(60.44 \pm 5.05)$ years old. The patient's information was similar and comparable.

Inclusion criteria: After various clinical examinations, the patient was diagnosed with cerebral thrombosis, cerebral infarction, cerebral hemorrhage and other diseases. The patients were informed and agreed to participate in the study.

Exclusion criteria: patients with mental disorders; patients with poor compliance; patients with other major diseases.

\subsection{Research methods}

After admission, patients should stop using all Chinese medicines and give them western medicine treatment. In the specific implementation process, the control group patients were mainly given Naofukang injection with a dose of $250 \mathrm{ml}$, and intervention was performed by 
intravenous infusion, once a day. The patients in the test group used Naofukang injection combined with Naomaitong oral liquid. The implementation method and dosage of Naofukang injection were the same as those of the control group. Naomaitong was added as an oral solution with a dose of $10 \mathrm{ml}$. It was taken 3 times a day. Patients were not given anti-platelet, anticoagulant, or fiber-promoting drugs. Both groups were treated continuously for 4 weeks.

\subsection{Statistical methods}

Using the statistical software SPSS20.0 as a tool and performing statistical analysis on the data presented in this study. Verifying the $T$ value of the comparison result of the measurement data $(\bar{x} \pm \mathrm{s})$, and verifying the $X^{2}$ value of the result of the count data (n, \%), when the result shows $\mathrm{P}$ $<0.05$, it means that the difference between the groups has statistical analysis value [3].

\section{Results}

\subsection{Treatment effect}

There were 2 patients in the test group who were ineffective with the treatment effective rate of $94.00 \%$, and 9 patients in the control group who were ineffective with the treatment effective rate of $82.00 \%$. The data between the groups was largely different, which was significant $(\mathrm{P}<0.05)$.

Table1. Treatment effect analysis of two groups of patients [n(\%)]

\begin{tabular}{|c|c|c|c|c|c|}
\hline Group & $\begin{array}{c}\text { Fully } \\
\text { recovered }\end{array}$ & Distinguished & Effective & Ineffective & Effective rate \\
\hline $\begin{array}{l}\text { Test group } \\
(\mathbf{n = 5 0})\end{array}$ & $30(60.00)$ & $10(20.00)$ & $7(14.00)$ & $3(6.00)$ & $47(94.00)$ \\
\hline $\begin{array}{l}\text { Control group } \\
\quad(\mathbf{n}=\mathbf{5 0})\end{array}$ & $20(40.00)$ & $11(22.00)$ & $10(20.00)$ & $9(18.00)$ & $41(82.00)$ \\
\hline $\begin{array}{c}\mathbf{X}^{2} \\
\mathbf{P}\end{array}$ & & & & & $\begin{array}{l}11.045 \\
<0.05\end{array}$ \\
\hline
\end{tabular}

\subsection{Comparison of plasma indexes}

Before treatment, the difference in FIB and PagT levels between the two groups was small, with no significant difference, $\mathrm{P}>0.05$. After intervention, the two plasma indexes of the patients both changed. By contrast, the improvement effect of the patients in the test group was more significant. The difference between the two groups of data was large, $\mathrm{P}<0.05$, which was significant.

Table2. Comparison of FIB and PagT levels before and after treatment between two groups of patients $( \pm \mathrm{s})$

\begin{tabular}{|c|c|c|c|c|}
\hline \multirow{2}{*}{ Group } & \multicolumn{2}{|c|}{ FIB（g/L ) } & \multicolumn{2}{|c|}{$\operatorname{PagT}(\%)$} \\
\hline & Before intervention & After intervention & Before intervention & After intervention \\
\hline $\begin{array}{c}\text { Test group } \\
(\mathbf{n}=\mathbf{5 0})\end{array}$ & $5.04 \pm 1.00$ & $3.95 \pm 1.85$ & $48.34 \pm 14.06$ & $41.35 \pm 8.34$ \\
\hline $\begin{array}{l}\text { Control group } \\
\quad(\mathbf{n}=\mathbf{5 0})\end{array}$ & $4.96 \pm 1.33$ & $4.76 \pm 0.55$ & $47.95 \pm 16.05$ & $45.05 \pm 8.55$ \\
\hline $\mathbf{t}$ & 0.586 & 4.697 & 0.445 & 5.045 \\
\hline $\mathbf{P}$ & $>0.05$ & $<0.05$ & $>0.05$ & $<0.05$ \\
\hline
\end{tabular}

\section{Conclusion}

In the context of the continuous development and construction of our society, people's living standards have been greatly improved, the pressure of life has also increased, and the diet structure has changed, resulting in the gradually increasing of incidence of hypertension, hyperlipidemia and hyperglycemia, which to a certain extent increased the incidence of cerebrovascular disease. The common diseased groups are middle-aged and elderly people. Cardio-cerebrovascular disease has the clinical characteristics of long course and easy to repeat. Once the disease occurs, the patient's quality of life, physical health and life safety will be greatly affected [4]. Therefore, for patients with cardiovascular and cerebrovascular diseases, medical staff need to adopt targeted treatment and clinical care, so as to achieve good clinical prevention and treatment effects.

Cerebrovascular disease has become the main factor leading to the disability and death of Chinese residents in the current development. Therefore, effective therapeutic intervention should be given to patients clinically. From the perspective of Chinese medicine theory, patients with cerebral infarction in the acute stage often show in the meridians and middle collateral. A small number of patients also exist in the middle organs and the abdomen. 
The main factor leading to the patient's morbidity is the blood circulation of qi. Qi deficiency leads to the accumulation of moisture and stagnation. Both of them produce sputum under the condition of accumulation of moisture, causing phlegm stagnation and coagulation, which causes obstruction of the blood vessel [5]. Therefore, the main factors leading to the patient's morbidity are qi, phlegm and stasis, which is also the key content of the treatment of this disease. Starting from the principle of the same treatment of different diseases in traditional Chinese medicine, although the patients have different types of diseases, the causes are mostly liver and kidney yin deficiency, liver yang forming wind syndrome, liver yang hyperactivity, liver wind internal movement and so on. Therefore, Naomaitong needs to be applied, mainly for nourishing yin, dampness and heat, nourishing liver and kidney and calming liver wind.

In terms of Naomaitong, the medicine is composed of traditional Chinese medicine, mainly including leeches, black snakes, safflowers, whole scorpions and gastrodia. Among them, safflowers can achieve the effect of circulation of blood stasis, smoothing the meridians, and improving the situation of patient's whole body congestion, and leeches can achieve the effect of eliminating blood stasis, and the application effect is significant in patients with congestion and stagnation of dead blood [6]. According to the results of modern pharmacology research, Naomaitong contains substances such as heparin and antithrombotic. At the same time, hirudin has a significant anticoagulant effect, and scorpion has a Tongluo analgesic effect, and its hemolytic effect is significant. The whole scorpion and trimethylamine scorpionate have a significant effect in sedation and anticonvulsant, and also a significant and long lasting anti-hypertensive effect. Black snake has effect of relaxing tendons and dredging collateral, according to the results of modern research, this drug can also achieve vasodilation. Among them, Gastrodia has the effect of calming the liver wind, in the case of sparse collaterals and analgesia, it can improve the patients' fistula weakness and numbness. The alkaloids can achieve sedation, analgesia, anticonvulsant and antiepileptic effects [7]. Mulberry parasite, eucommia ulmoides and wolfberry have significant effects on liver and kidney supplementation and strengthening muscles and bones. After completing the patient's treatment, it is necessary to proceed from the patient's actual situation and give the patient three stages of care to improve the patient's adverse psychological conditions, and to improve the effectiveness rate of clinical treatment. From the perspective of prevention of cardiovascular and cerebrovascular diseases, it can increase the importance of medical staff on the prevention of cardiovascular and cerebrovascular diseases, so as to avoid the recurrence and aggravation of the disease, so as to help patients recover faster. At the same time, the medical staff can also help patients develop good eating, exercise, and lifestyle habits to ensure that patients maintain a healthy diet and adequate exercise [8]. From the perspective of clinical treatment, it can be combined with the patient's clinical symptoms and the severity of the disease, and can be targeted to the patients, through drug and intravenous treatment, to alleviate the patient's clinical symptoms and avoid further deterioration of the disease, so as to achieve good therapeutic effect [9]. After the application of Naomaitong, it can achieve the effects of anti-infection, blood circulation, blood stasis reduction, lipid-lowering and improvement of heart function. After giving patients intervention in this study, the results showed that the patients in the test group had a higher effective rate, and the patients' FIB and PagT levels after treatment were lower, which has a significant effect.

In summary, cerebrovascular disease has a greater impact on the health of patients, and effective nursing interventions need to be given to patients. When Naomaitong is applied, the intervention effect of patients is significant, and the plasma FIB and PagT levels of patients can be reduced, which has a positive meaning for clinical development, and it can be used and promoted in clinical treatment.

\section{Acknowledgement}

I sincerely thank my mentor, for the guidance of the ideas proposed by me in the process of writing this paper, and his careful review during the creation and completion of the paper. Secondly, I would like to thank all the mentors who have taught me, it is your careful teaching that has enabled me to have a deeper understanding of professional knowledge and ensure the completion of this paper. Finally, I would like to thank the predecessors and scholars for their rich knowledge, experience and research results, which provided theoretical support and help for the writing of this paper.

\section{References}

1. Yimin Liu, "Analysis of the clinical effect of Naomaitong in treating cerebrovascular diseases"[J]. Chinese Pharmaceutical Economics, 2013(01):207208.

2. Qingxun Hou, "Self-made Naomaitong for the treatment of 114 cases of cerebrovascular diseases" [J]. Practical Journal of Internal Medicine, 2008(10): 22-23.

3. Luyi Luo, Runfen Feng, Renbin Qiu, Mei Luo, Huanbin Yang, Pengyi Chen, "Clinical observation of Naomaitong in treating cerebral arteriosclerosis" [J]. Hebei Journal of Traditional Chinese Medicine, 2005(01): 7-8.

4. Jianhua Sun, "Clinical research report of cerebrovascular disease" [J]. Practical CardioCerebro-Pulmonary Vascular Disease, 2014,22(02):159-160.

5. Yongxiang Gao, Farong Zhang, Honghui Sun, Ming Lei, Wanhui Cao, "Effects of Naomaitong Injection on Tumor Necrosis Factor and Calcitonin GeneRelated Peptide in Brain Tissue of Rats with Ischemic Stroke" [J]. Journal of Chengdu University of Traditional Chinese Medicine, 2001 (01): 38-39+44.

6. Yingchun Zhou, "Clinical and experimental study of Naomaitong in the treatment of acute ischemic stroke" 
[J]. Journal of Shandong University of Traditional Chinese Medicine, 1996(02) 94-99.

7. Mulin $\mathrm{Xu}$, Ruhai Cheng, "Clinical observation of Naomaitong in treating 228 cases of cerebral infarction" [J]. Tianjin University of Traditional Chinese Medicine, 1996(06): 12-13+15.

8. Yingchun Zhou, Nianfang Shao, Zhaoping Liu, "Experimental study of Naomaitong oral liquid in the treatment of acute ischemic stroke"[J]. Journal of Guangzhou University of Traditional Chinese Medicine, 1999(04):268-271.

9. Guoxiang Liu, Hong Yu, Shubao Ying, Songshi Li, Lisheng Wang, Wenying Zhi, "Observation on the therapeutic effect of 100 cases of ischemic cardiocerebrovascular disease with Chinese medicine "Xinnaomaitong" oral liquid" [J]. Shizhen Chinese Medicine Research, 1991(03): 106-108. 